LETTERS TO THE EDITOR

\section{Alcohol consumption in the UK armed forces: are we drinking too much?}

The article, 'A pilot study of an Enhanced Mental Health Assessment during routine and discharge medicals in the British Armed Forces' ${ }^{1}$ published in this edition of the JR Army Med Corps, produced some interesting results regarding alcohol consumption in the armed forces, which we would like to discuss in more detail.

The UK military has a historical relationship with alcohol. ${ }^{2}$ One recent study found that the 'prevalence of alcoholrelated harm and alcohol dependence within the UK Armed Forces is greater than in the general population'. ${ }^{3}$ This study and several others have supported the belief that military personnel consume more alcohol than civilians when using comparable populations. ${ }^{45}$ Alcohol consumption is part of the UK military culture and may foster social cohesion when consumed in moderation. ${ }^{6}$ However, the health implications of excessive alcohol consumption are significant. Iversen et al noted that alcohol misuse was often comorbid with neurotic problems in the UK military ${ }^{7}$ while Verrall found that when consuming alcohol at a risky level, there could be adverse physical, mental and psychological health outcomes associated with combat exposure. ${ }^{2}$ In addition, the media mainly focus on post-traumatic stress disorder as an adverse mental health outcome from military service, whereas the reality is that the most frequent mental health problems for veterans are alcohol use disorders, depression and adjustment disorders. ${ }^{8}$

Our pilot study ${ }^{1}$ used a questionnaire during routine and discharge medicals at four military medical centres and specifically included three questions from the Alcohol Use Disorder Identification Test Consumption (AUDIT-C), ${ }^{9}$ a validated alcohol misuse screening tool where a total score of 5 or more indicates higher risk drinking; it is however noteworthy that validation data of the AUDIT-C within a military population have not been published. The results from the pilot study revealed that $65 \%$ of service personnel had an AUDIT-C score $>4$, indicating 'higher risk drinking' (Figure 1).

We analysed correlations among the AUDIT-C scores, frequency of drinking alcohol and identification of possible

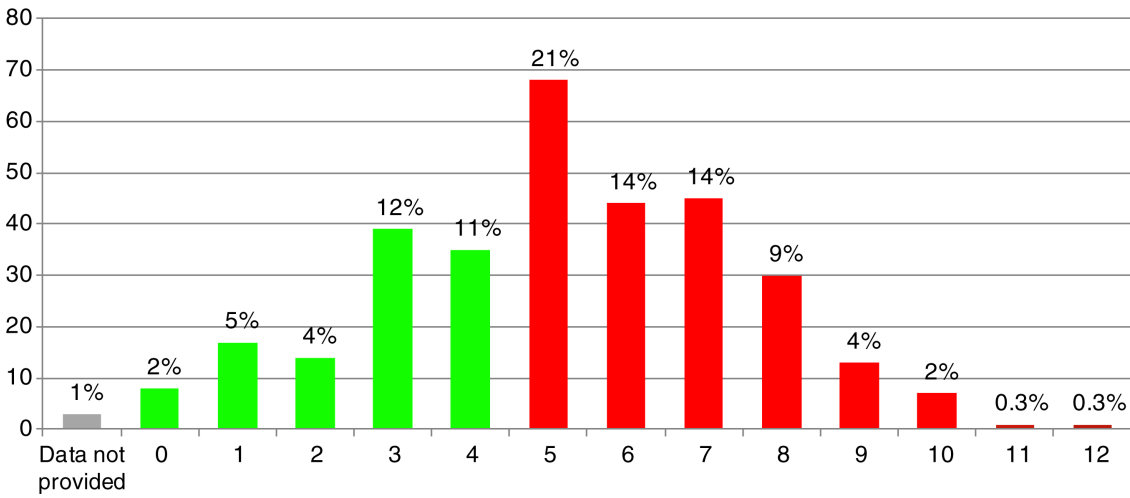

Figure 1 Alcohol Use Disorder Identification Test (AUDIT-C) scores. Percentages of patients scoring $0-12$. A score of 5 or higher $(65 \%)$ indicates higher risk drinking.

mental health problems using standard brief survey tools for depression, anxiety, post-traumatic stress disorder, sleep problems and irritability. We found that those personnel with more risky drinking habits were more likely to be identified as having possible mental health problems. Figure 2 demonstrates that personnel with an AUDIT-C score of $\geq 5 \quad(n=237)$ were identified as having possible mental health problems more frequently $(11.8 \%$ vs $7.4 \%)$ than those scoring 4 or less $(n=122)$. In addition, we found that the 45 personnel who reported drinking alcohol more than four times per week were twice as likely to report positive scores on the brief mental health survey tools than those who did not (20\% vs $10 \%)$.

As part of the Enhanced Mental Health Assessment (EMHA) process, all those identified as having potential mental health problems, including risky alcohol use, were offered assistance. As a minimum, these patients were offered health and lifestyle advice. The EMHA

could therefore be viewed as a form of screening and brief interventions (SBI). SBI for alcohol misuse disorders have been shown to be effective in primary care in non-military populations. ${ }^{10}{ }^{11}$ With the addition of a formalised brief intervention, the EMHA may offer an opportunity to improve the drinking habits and, as demonstrated in our paper, ${ }^{1}$ the mental health of the armed forces population in the UK.

\section{SO, WHAT ARE THE IMPLICATIONS FOR CLINICIANS?}

A score of 5 of more on AUDIT-C suggests possible risky alcohol consumption and should lead to completion of the full 10 question AUDIT questionnaire (AUDIT-C comprises of three questions. AUDIT is a 10-part questionnaire). A score of 8 or more on AUDIT confirms hazardous alcohol consumption as very likely to be present. A score of 16 or more is highly suggestive of harmful alcohol use (ICD-10 code F10.1).

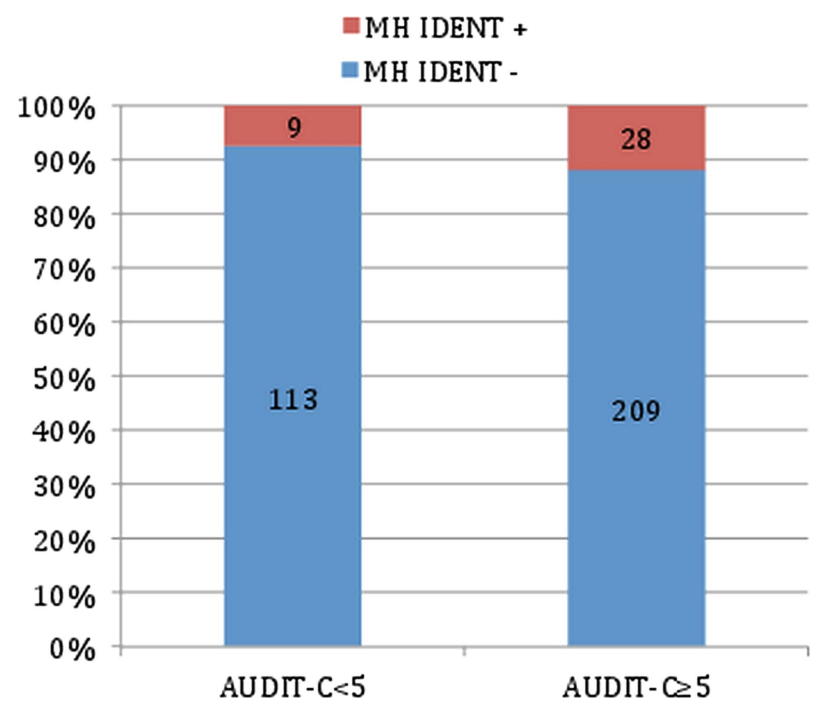

Figure 2 Identification of potential mental health problems related to Alcohol Use Disorder Identification Test (AUDIT-C) scores. 
Hazardous alcohol consumption requires a brief intervention, which can be as simple as a few minutes of discussion about potential harm of alcohol use and/ or provision of a patient information leaflet. Good medical practice would be to review several weeks later to ascertain whether the person has altered his/her behaviour. Harmful alcohol use warrants the same, as well as a full mental health assessment, as comorbid mental health conditions will be common. It is also important to consider any occupational risks of alcohol misuse. Once a full assessment has been carried out, the clinician must discuss with the patients what they would like to do about their alcohol misuse. A referral to Defence Community Mental Health should be considered in these cases.

Marcela Aguirre, ${ }^{1} \mathrm{~N}$ Greenberg, ${ }^{2} \mathrm{~J}$ Sharpley, ${ }^{3}$ R Simpson, ${ }^{1}$ C Wall ${ }^{3}$

${ }^{1}$ Department of General Practice, Royal Centre for Defence Medicine, Birmingham, UK

${ }^{2}$ Academic Centre for Defence Mental Health, Weston Education Centre, London, UK

${ }^{3}$ Department of Community Mental Health, HM Naval Base, Portsmouth, UK

Correspondence to Capt M Aguirre, Royal Centre for Defence Medicine, ICT Research Park, Birmingham, Vincent Drive, B15 2SQ, UK; m.aguirre@doctors.org.uk

Acknowledgements We would like to thank Brigadier Cordell (DMS Head of Medical Strategy and Policy) for his support throughout this project. We would also like to thank Michael Rowe (Military Medical Librarian, Defence Medical Library Services) for his support with literature searches and background research, Amarjit Samra (Director of Research, JMC Medical Directorate) for invaluable advice on conducting research and Anna Brown (Operations Manager, JMC Medical Directorate) for support with data analysis.

Contributors MA drafted the manuscript. However, every author contributed to the writing of the manuscript, the data analysis and the charts.

Competing interests None.

Patient consent Obtained.

Provenance and peer review Not commissioned; internally peer reviewed.

To cite Aguirre M, Greenberg N, Sharpley J, et al. J R Army Med Corps 2014;160:72-73.

Received 7 March 2013

Accepted 9 March 2013

Published Online First 17 June 2013

J R Army Med Corps 2014;160:72-73.

doi:10.1136/jramc-2013-000059

\section{REFERENCES}

1 Aguirre M, Greenberg N, Sharpley J, et al. A pilot study of an Enhanced Mental Health Assessment during routine and discharge medicals in the British Armed Forces. J R Army Med Corps 2014;160:15-9.

2 Verrall NG. A review of military research into alcohol consumption. J R Army Med Corps 2011; 157:164-9.
3 Fear NT, Iversen A, Meltzer $\mathrm{H}$, et al. Patterns of drinking in the UK Armed Forces. Addiction 2007:102:1749-59.

4 Hooper R, Rona J, Jones M, et al. Cigarette and alcohol use in the UK Armed Forces, and their association with combat exposures: A prospective study. Addict Behav 2008;33:1067-71.

5 Henderson A, Langston V, Greenberg N. Alcohol misuse in the Royal Navy. Occup Med 2009;59:25-31.

6 Du Preez J, Sundin J, Wessely S, et al. Unit cohesion and mental health in the UK armed forces. Occup Med 2012;62:47-53.

7 Iversen $A C$, van $\mathrm{L}$, Hughes $\mathrm{JH}_{\text {, }}$ et al. The prevalence of common mental disorders and PTSD in the UK military: using data from a clinical interview-based study. BMC Psychiatry 2009;9:68.

8 Murphy D, Iversen A, Greenberg N. The mental health of veterans. J $R$ Army Med Corps 2008; 154:136-9.

9 Bush K, Kivlahan DR, McDonell MB, et al. The AUDIT alcohol consumption questions (AUDIT-C): an effective brief screening test for problem drinking. Ambulatory Care Quality Improvement Project (ACQUIP). 10. Alcohol Use Disorders Identification Test. Arch Intern Med 1998;158:1789-95.

10 Purshouse R, Brennan A, Rafia R, et al. Modelling the Cost-Effectiveness of Alcohol Screening and Brief Interventions in Primary Care in England. Alcohol Alcohol 2012;2:180-8.

11 Jackson R, Johnson M, Campbell F, et al. Screening and brief interventions for prevention and early identification of alcohol use disorders in adults and young people. Sheffield: University of Sheffield, School of Health and Related Research (ScHARR) Public Health Collaborating Centre, 2010. 